\title{
Homomorphisms of planar signed graphs to signed projective cubes
}

\author{
Reza Naserasr $^{1}$ and Edita Rollováa ${ }^{2}$ and Éric Sopena $a^{3,4}$ \\ ${ }^{1}$ CNRS, LRI, UMR8623, Univ. Paris-Sud 11, F-91405 Orsay Cedex, France \\ ${ }^{2}$ Department of Mathematics and Institute of Theoretical Computer Science, University of West Bohemia, Univerz- \\ itní 8, 306 14, Plzeň, Czech Republic \\ ${ }^{3}$ Univ. Bordeaux, LaBRI, UMR5800, F-33400 Talence, France \\ ${ }^{4}$ CNRS, LaBRI, UMR5800, F-33400 Talence, France
}

\begin{abstract}
We conjecture that every signed graph of unbalanced girth $2 g$, whose underlying graph is bipartite and planar, admits a homomorphism to the signed projective cube of dimension $2 g-1$. Our main result is to show that for a given $g$, this conjecture is equivalent to the corresponding case $(k=2 g)$ of a conjecture of Seymour claiming that every planar $k$-regular multigraph with no odd edge-cut of less than $k$ edges is $k$-edge-colorable. To this end, we exhibit several properties of signed projective cubes and establish a folding lemma for planar even signed graphs.
\end{abstract}

Keywords: homomorphism, planar signed graph, projective cube, signed graph

\section{Introduction}

It is a classic result of Tait from 1890 that the Four-Color Theorem (Conjecture at that time) is equivalent to the statement that every cubic bridgeless planar graph is 3-edge-colorable. An extension of this equivalent statement was proposed as a conjecture using the notion of an odd cut, that is a partition $(X, Y)$ of the set of vertices where $|X|$ is odd. It is easily observed that if a $k$-regular multigraph is $k$-edge-colorable, then the number of edges with exactly one end in $X$, assuming $|X|$ is odd, is at least $k$. Seymour conjectured in 1975 that for planar multigraphs the converse is also true, which generalizes Tait's statement:

Conjecture 1.1 (Seymour [13]) Every $k$-regular planar multigraph with no odd edge-cut of less than $k$ edges is k-edge-colorable.

A direct extension of the Four-Color Theorem, using the language of graph homomorphisms, was introduced in [10] where it was shown that this conjecture is essentially equivalent to Seymour's conjecture for odd values of $k$. In an unpublished manuscript [5], B. Guenin, after introducing the notion of signedgraph homomorphisms, provided a further extension of this conjecture and the Four-Color Theorem. He has then shown relations between his conjecture and several other conjectures.

The theory of homomorphisms of signed graphs includes in particular the theory of graph homomorphisms. A first paper on a comprehensive study of this notion was recently written by the authors of subm. to DMTCS (c) by the authors Discrete Mathematics and Theoretical Computer Science (DMTCS), Nancy, France 
this work. Here we would like to emphasis on a direct extension of the Four-Color Theorem and its relation with Seymour's conjecture. We introduce the basic notations but we refer to [12] and references mentioned there for more details.

Given a graph $G$, a signature on $G$ is a mapping that assigns to each edge of $G$ either a positive or a negative sign. A signature is normally denoted by the set $\Sigma$ of negative edges. Given a signature $\Sigma$ on a graph $G$, resigning at a vertex $v$ is to change the sign of each edge incident with $v$. Two signatures $\Sigma_{1}$ and $\Sigma_{2}$ on $G$ are equivalent if one can be obtained from the other by a sequence of resignings or, equivalently, by changing the signs of the edges of an edge-cut. A graph $G$ equipped with a signature $\Sigma$ is a signified graph, denoted $(G, \Sigma)$. A signed graph is a maximal class of signified graphs, all of whose signatures are equivalent. For convenience, a signed graph will also be denoted $(G, \Sigma)$ where $\Sigma$ is any member of the class of equivalent signatures.

An important notion in signed graphs is the following. An unbalanced cycle in a signed graph $(G, \Sigma)$ is a cycle having an odd number of negative edges. Note that this is independent of the choice of a representative signature. Furthermore, the notion of unbalanced cycle is, in some sense, an extension of the classic notion of an odd cycle (a cycle with odd length), as a cycle of $(G, E(G))$ is unbalanced if and only if it is an odd cycle of $G$. The unbalanced-girth of $(G, \Sigma)$ is then the shortest length of an unbalanced cycle of $(G, \Sigma)$. A cycle that is not unbalanced, i.e., a cycle that has an even number of negative edges (possibly none), is called balanced.

Note that if a signed Eulerian graph contains an odd number of negative edges, it must contain an unbalanced cycle. Therefore, if $W$ is a closed walk in $G$ with an odd number of negative edges in $(G, \Sigma)$, then the subgraph induced by the edges of $W$ contains an unbalanced cycle.

One of the first theorems in the theory of signed graphs is that the set of unbalanced cycles (equivalently the set of balanced cycles) uniquely determines the associated class of signatures. More precisely:

Theorem 1.2 (Zaslavsky [14]) Two signatures $\Sigma_{1}$ and $\Sigma_{2}$ on a graph $G$ are equivalent if and only if they induce the same set of unbalanced cycles.

An important subclass of signed graphs, called consistent signed graphs, is the class of signed graphs whose balanced cycles are all of even length and whose lengths of unbalanced cycles are all of the same parity. This class itself consists of two parts. When all the unbalanced cycles are of odd length, then the set of unbalanced cycles of $(G, \Sigma)$ is exactly the set of odd-length cycles of $G$, thus in this case, by Theorem 1.2, $E(G)$ is a signature and $(G, \Sigma)=(G, E(G))$. Such a signed graph will then be called an odd signed graph. When the lengths of all balanced and unbalanced cycles are even, the graph $G$ must be bipartite, and $\Sigma$ can be any subset of edges. Such a signed graph will be called a signed bipartite graph.

Given two graphs $G$ and $H$, a homomorphism of $G$ to $H$ is a mapping $\phi: V(G) \rightarrow V(H)$ such that if $x y \in E(G)$ then $\phi(x) \phi(y) \in E(H)$. We denote by $G \rightarrow H$ the existence of a homomorphism of $G$ to $H$. This notion extends the notion of coloring because a graph $G$ is $k$-colorable if and only if $G \rightarrow K_{k}$. Given two signed graphs $\left(G_{1}, \Sigma_{1}\right)$ and $\left(G_{2}, \Sigma_{2}\right)$ we say that $\left(G_{1}, \Sigma_{1}\right)$ admits a signed-graph homomorphism, or homomorphism for short, to $\left(G_{2}, \Sigma_{2}\right)$ if there are signatures $\Sigma_{1}^{\prime}$ and $\Sigma_{2}^{\prime}$ equivalent to $\Sigma_{1}$ and $\Sigma_{2}$, respectively, and a homomorphism $\varphi$ of $G_{1}$ to $G_{2}$ such that $\varphi$ also preserves the signs of edges given by $\Sigma_{1}^{\prime}$ and $\Sigma_{2}^{\prime}$. It is easily observed that the existence of $\varphi$ is independent of the choice of the signature in the image graph while the choice of the signature of $\left(G_{1}, \Sigma_{1}^{\prime}\right)$ is essential. Thus the binary relation $\left(G_{1}, \Sigma_{1}\right) \rightarrow\left(G_{2}, \Sigma_{2}\right)$, which denotes the existence of a homomorphism of $\left(G_{1}, \Sigma_{1}\right)$ to $\left(G_{2}, \Sigma_{2}\right)$, is transitive. 
For every integer $k \geq 3$, we denote by $U C_{k}$ the unbalanced cycle of length $k$, that is $U C_{k}=\left(C_{k},\{e\}\right)$, where $e$ is any edge of the cycle $C_{k}$. One of the first results in the theory of signed-graph homomorphisms is the following easy-to-prove lemma.

Lemma 1.3 There is a homomorphism of $U C_{k}$ to $U C_{\ell}$ if and only if $k \geq \ell$ and $k \equiv \ell(\bmod 2)$.

Another key notion for this work is the notion of minors. A minor of a signed graph $(G, \Sigma)$ is a signed graph obtained from $(G, \Sigma)$ by a sequence of $(i)$ deleting vertices or edges, $(i i)$ contracting positive edges and ( iii $)$ resigning, in any order. In particular, this notion allows to express in terms of (odd) signed graphs the following conjecture, proposed by Gerards and Seymour (see [7], p. 115), which extends the celebrated Hadwiger's Conjecture.

Conjecture 1.4 (Odd Hadwiger's Conjecture) If $(G, E(G))$ does not have $\left(K_{n}, E\left(K_{n}\right)\right)$ as a minor, then $\chi(G) \leq n-1$.

Using the definition of signed projective cube from the next section, the following conjecture is the main concern of this work:

Conjecture 1.5 Every consistent planar signed graph of unbalanced girth $k$ admits a homomorphism to the signed projective cube of dimension $k-1$.

The case $k=3$ of this conjecture is indeed the Four-Color Theorem. It is proved in [10] that this is equivalent to Conjecture 1.1 for every odd $k$. Here we do the analog for even values of $k$, i.e. for planar signed bipartite graphs, and prove the following:

Theorem 1.6 The following two statements are equivalent:

(i) Every planar $2 k$-regular multigraph with no odd edge-cut of less than $2 k$ edges is $2 k$-edge-colorable.

(ii) Every planar signed bipartite graph of unbalanced girth at least $2 k$ admits a homomorphism to the signed projective cube of dimension $2 k-1$.

To this end we prove an analog of the "folding lemma" from [9] for the class of planar signed bipartite graphs. We note that, as it is shown in [12], the restriction of the notion of signed-graph homomorphism to the class of signed bipartite graphs already captures the notion of graph coloring and graph homomorphism through simple and natural graph operations. Roughly speaking, we can associate a signed bipartite graph $\left(S(G), E_{S}(G)\right)$ with any graph $G$ such that $(i) \chi(G) \leq k$ if and only if $\left(S(G), E_{S}(G)\right) \rightarrow\left(K_{k, k}, M_{k}\right)$, where $M_{k}$ is any perfect matching of the complete bipartite graph $K_{k, k}$, and $(i i)$ for any graphs $G$ and $H, G \rightarrow H$ if and only if $\left(S(G), E_{S}(G)\right) \rightarrow\left(S(H), E_{S}(H)\right)$.

The structure of the paper is as follows: in the next section we define the signed projective cubes and prove their main properties. Then we prove the folding lemma for planar signed bipartite graphs in Section 3 and Theorem 1.6 in Section 4.

\section{Signed Projective Cubes}

Recall that for $d \geq 2$, the hypercube of dimension $d$, denoted $\mathcal{H}_{d}$, is the graph with vertex set $\left(\mathbb{Z}_{2}\right)^{d}$, two vertices $x$ and $y$ being adjacent if $x-y \in\left\{e_{1}, e_{2}, \ldots, e_{d}\right\}$, where $e_{i}$ is the vector of $\left(\mathbb{Z}_{2}\right)^{d}$ with the $i$-th coordinate being 1 and other coordinates being 0 . This can be seen as the skeleton of the geometric hypercube, or as a discrete version of the $d$-dimensional sphere. The distance between any two vertices 

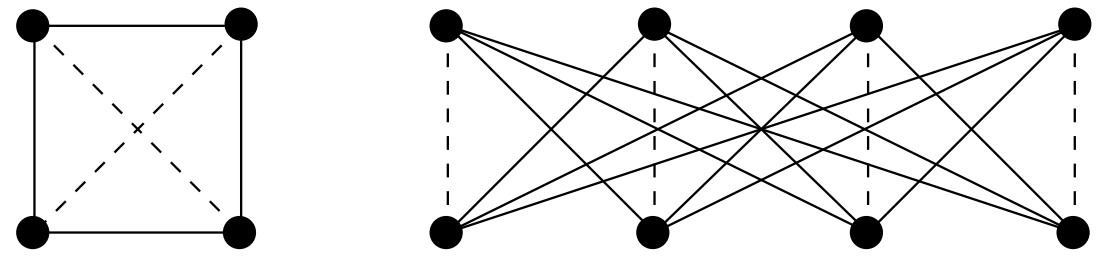

Fig. 1: Signed projective cubes of dimension 2 and 3

in $\mathcal{H}_{d}$ is thus the number of coordinates in which they differ. Two vertices in $\mathcal{H}_{d}$ are said to be antipodal if they are at maximum graph distance. Hence, each vertex $v$ has a unique antipode $v+J$, where $J=$ $(1,1, \ldots, 1)$, whose distance from $v$ is $d$.

Equivalenlty, the hypercube $\mathcal{H}_{d}$ is inductively obtained from two disjoint copies of $\mathcal{H}_{d-1}$ by adding an edge between each pair of corresponding vertices in the two copies. In this view, to obtain the antipodal of a vertex $x$ in $\mathcal{H}_{d}$ we must first find its antipodal $x^{*}$ in the copy of $\mathcal{H}_{d-1}$ to which $x$ belongs. Then the twin of $x^{*}$ in the other copy is the antipodal of $x$ in $\mathcal{H}_{d}$.

Projective cubes can be defined in several ways, our first definition is the one that justifies their name. Just as the projective space of dimension $d$ is built from the sphere of dimension $d+1$, we define the projective cube of dimension $d$, denoted $\mathcal{P C}$, to be the homomorphic image of $\mathcal{H}_{d+1}$ under the identification of antipodal pairs. If we consider two copies of $\mathcal{H}_{d}$ which are the building blocks of $\mathcal{H}_{d+1}$, the above mentioned projection will map vertices from one copy to another, where adjacencies are also preserved but the edges of the matching connecting one copy to another will become edges connecting each vertex of $\mathcal{H}_{d}$ to its antipodal in $\mathcal{H}_{d}$. Thus $\mathcal{P} \mathcal{C}_{d}$ can also be defined as the graph obtained from $\mathcal{H}_{d}$ by adding a new edge between each pair of antipodal vertices in $\mathcal{H}_{d}$. Since in the algebraic definition of $\mathcal{H}_{d}$ two vertices are antipodal if and only if their difference is $J$, we can also define $\mathcal{P C}$ as a Cayley graph as follows: $\mathcal{P} \mathcal{C}_{d}$ is the graph with vertex set $\left(\mathbb{Z}_{2}\right)^{d}$, where vertices $u$ and $v$ are adjacent if $u-v \in\left\{e_{1}, e_{2}, \ldots, e_{d}\right\} \cup\{J\}$. We will consider that such an edge $u v$ is labeled by $u-v$. We will also use the following:

Observation 2.1 For every $d \geq 2$, the sum of the edge labels of any cycle in $\mathcal{P C}_{d}$ is 0.

It is easy to check that $\mathcal{P C _ { 2 }}, \mathcal{P C}_{3}$ and $\mathcal{P C}$ are isomorphic to $K_{4}, K_{4,4}$ and the well-known Clebsch graph, respectively.

Using the Cayley definition of $\mathcal{P} \mathcal{C}_{d}$, let $\mathcal{J}$ be the set of edges labeled by $J$. We define the signed projective cube of dimension $d$, denoted $\mathcal{S P C}$, to be the signed graph $(\mathcal{P C}, \mathcal{J})$. The first two signed projective cubes are presented in Fig. 1. The presentation of $\mathcal{P C}$, given in Fig. 2, also shows the method of construction of the projective cubes. In these figures, dashed edges are negative and solid edges are positive.

We will first prove that $\mathcal{S P C}$ is a consistent signed graph and determine its unbalanced girth.

Theorem 2.2 All balanced cycles of $\mathcal{S P \mathcal { C } _ { d }}$ are of even length, all unbalanced cycles of $\mathcal{S P C}$ are of the same parity, and the unbalanced girth of $\mathcal{S P C}_{d}$ is $d+1$. Furthermore, for each unbalanced cycle $U C$ of $\mathcal{S P C}_{d}$ and for each $x \in\left\{e_{1}, e_{2}, \ldots, e_{d}\right\} \cup\{J\}$, there is an odd number of edges of UC labeled by $x$.

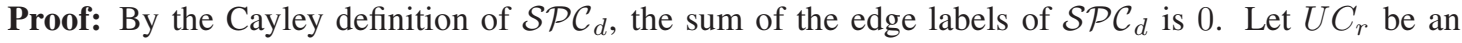
unbalanced cycle of length $r$ in $\mathcal{S P C}$. Thus, by the definition of an unbalanced cycle, there is an odd 


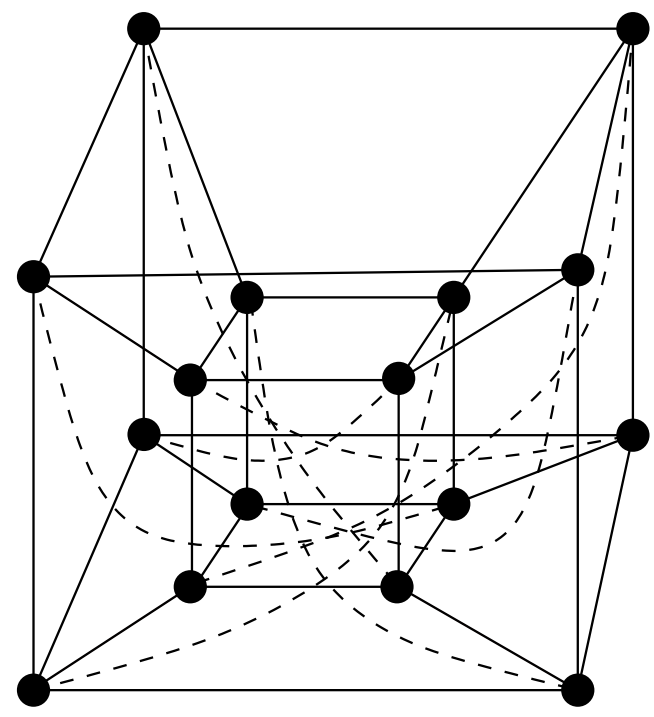

Fig. 2: Signed projective cube of dimension 4

number of edges in $U C_{r}$ labeled by $J$. To sum up the edge labels of $U C_{r}$ to 0 , each $e_{i}, i=1,2, \ldots, d$, must also appear an odd number of times. Thus $r \geq d+1$ and $r \equiv d+1(\bmod 2)$. In particular, this implies that the lengths of all the unbalanced cycles of $\mathcal{S P C}$ have the same parity.

Similarly, if $C$ is a balanced cycle then $C$ contains an even number of edges labeled by $J$, by definition. Since, by Observation 2.1, the sum of the edge labels on each cycle is 0 , there should be an even number of edges labeled by each of the $e_{i}$ 's. Therefore, each balanced cycle is of even length.

To see that $\mathcal{S P \mathcal { C } _ { d }}$ is actually of unbalanced girth $d+1$, note that an unbalanced cycle of length $d+1$ is induced by the following sequence of vertices: $v_{0}=(0, \ldots, 0), v_{i}=v_{i-1}+e_{i}$ for $1 \leq i \leq d$.

Corollary 2.3 The signed projective cube $\mathcal{S P C}_{2 d}$ is equivalent to $\left(\mathcal{P C} \mathcal{C}_{2 d}, E\left(\mathcal{P C}_{2 d}\right)\right)$.

Proof: By Theorem 2.2 a cycle in $\left(\mathcal{P C} \mathcal{C}_{2 d}, \mathcal{J}\right)$ is unbalanced if and only if it is of odd length. This is exactly the set of unbalanced cycles of $\left(\mathcal{P C}_{2 d}, E\left(\mathcal{P C} \mathcal{C}_{2 d}\right)\right)$. Hence, by Theorem $1.2,\left(\mathcal{P C} \mathcal{C}_{2 d}, \mathcal{J}\right)$ and $\left(\mathcal{P C}_{2 d}, E\left(\mathcal{P C} \mathcal{C}_{2 d}\right)\right)$ are equivalent.

A direct proof of this corollary (using resigning) is worth mentioning: for each $i, 1 \leq i \leq d$, the set of edges of $\mathcal{P} \mathcal{C}_{d}$ labeled either by $e_{i}$ or by $J$ forms an edge-cut $(X, Y)$ where $X$ is the set of vertices with $i$-th coordinate being 0 and $Y$ is the set of vertices with $i$-th coordinate being 1 . If for each such edge-cut we resign all the edges of the cut (by resigning at all the vertices of $X$ ), then each edge corresponding to an $e_{i}$ will have a negative sign (as it will be resigned only once) and each edge corresponding to $J$ will be resigned $d$ times, so that its sign would return to original negative if and only if $d$ is even. Note that through this process we have resigned at some vertices more than once. At the end, we have resigned at vertices with an odd number of coordinates being 0 .

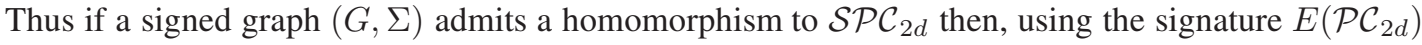

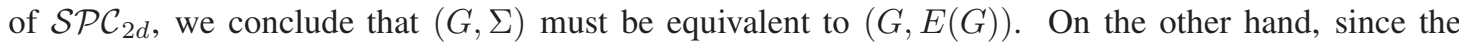


underlying graph of $\mathcal{S P C} \mathcal{C}_{2 d+1}$ is bipartite, if $(G, \Sigma)$ maps to $\mathcal{S P C} \mathcal{C}_{2 d+1}$, then $G$ must also be bipartite. Thus, in general, consistent signed graphs are the only graphs that can map to signed projective cubes. The following theorem shows that the problem of finding a mapping of a consistent signed graph to a signed projective cube is equivalent to a packing problem.

Theorem 2.4 A signed bipartite graph (resp. odd signed graph) admits a homomorphism to $\mathcal{S P C}_{2 d-1}$ $\left(\right.$ resp. $\mathcal{S P C}_{2 d}$ ) if and only if it admits at least $2 d-1$ (resp. $2 d$ ) edge-disjoint signatures.

Theorem 2.4 in this form first appeared in [5]. For even dimensions, i.e., for the case in brackets, since all edges being negative is a signature of $\mathcal{S P C}_{2 d}$, the problem of finding a homomorphism of $(G, \Sigma)$ to $\mathcal{S P \mathcal { C } _ { 2 d }}$ is reduced to the problem of finding a homomorphism of $G$ to $\mathcal{P C}$. Here, we give an independent proof for odd dimensions. Our proof can be easily adapted for even dimensions as well.

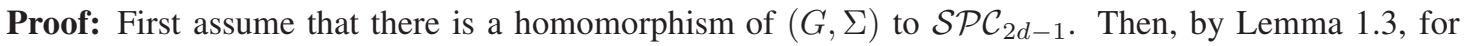
each unbalanced cycle $U C$ of $(G, \Sigma)$, there should be an unbalanced cycle in its image in $\mathcal{S P C}_{2 d-1}$. Furthermore, for each $e_{i}$, the set of edges of $U C$ that are mapped to an edge with label $e_{i}$, should be of odd size. On the other hand, for a balanced cycle $C$ of $(G, \Sigma)$ the set of edges of $C$ that are mapped to an edge with label $e_{i}$ should be of even size. Therefore, for each $e_{i}$ the set $E_{i}(G)$ of edges of $G$ which are mapped to edges of $\mathcal{S P} \mathcal{C}_{2 d-1}$ with label $e_{i}$ has the property that its intersection with each balanced (resp. unbalanced) cycle of $(G, \Sigma)$ is of even (resp. odd) size. Thus, by Theorem 1.2, $E_{i}(G)$ is equivalent to $\Sigma$ and obviously the $E_{i}(G)$ 's are edge disjoint.

For the converse, suppose $E_{1}, E_{2}, \ldots, E_{2 d-1}$ are sets of edge-disjoint signatures equivalent to $\Sigma$ and let $\hat{E}=E_{1} \cup E_{2}, \cup \cdots \cup E_{2 d-1}$.

We first claim that $E_{J}=E-\hat{E}$ is also a signature. We use Theorem 1.2 to prove this. If $U C$ is an unbalanced cycle of $(G, \Sigma)$, then it contains an odd number of edges from each $E_{i}, 1 \leq i \leq 2 d-1$ and, therefore, it contains an odd number of edges from $\hat{E}$. Since $U C$ is of even length, it has an odd number of edges from $E_{J}=E-\hat{E}$. Now, let $C$ be a balanced cycle of $(G, \Sigma)$. Clearly, the intersection of $C$ with each $E_{i}, 1 \leq i \leq 2 d-1$, and hence with $\hat{E}$, contains an even number of edges. Again, since $C$ has an even number of edges, the intersection of $C$ with $E_{J}=E-\hat{E}$ also has an even number of edges. Therefore, the set of unbalanced cycles of $(G, \Sigma)$ is exactly the set of cycles whose intersection with $E_{J}$ contains an odd number of edges.

Let now $\varphi: E(G) \rightarrow\left\{e_{1}, e_{2}, \ldots, e_{2 d-1}\right\} \cup\{J\}$ be defined as follows: if $u v \in E_{i}, 1 \leq i \leq 2 d-1$, then $\varphi(u v)=e_{i}$ and if $u v \in E_{J}$, then $\varphi(e)=J$. It is easy to verify now that given a cycle $C$ of $G$, $\sum_{u v \in E(C)} \varphi(u v)$ is 0 (in $\left.\left(\mathbb{Z}_{2}\right)^{2 d-1}\right)$.

A homomorphism of $(G, \Sigma)$, using its representation $\left(G, E_{J}\right)$, to $\mathcal{S P} \mathcal{C}_{2 d-1}$ can be now built as follows: for each connected component $G^{\prime}$ of $G$ choose a vertex $x$ and let $\phi(x)=0$. Then for any other vertex $y$ choose a path $P$ with $x$ and $y$ being its two ends and let $\phi(y)=\sum_{u v \in E(P)} \varphi(u v)$. Since $\varphi$ adds up to

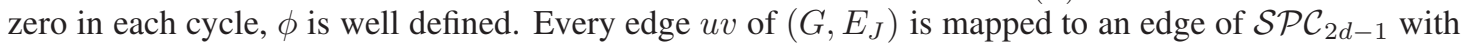
label $\phi(v)-\phi(u)$ and it is easy to check that $\phi$ is a homomorphism of $\left(G, E_{J}\right)$ to $\mathcal{S P C}_{2 d-1}$.

As an easy corollary we get that the homomorphism relation between signed projective cubes themselves is very much like that of the homomorphism relation between cycles as given in Lemma 1.3:

Theorem 2.5 There is a homomorphism of $\mathcal{S P C} \mathcal{C}_{d}$ to $\mathcal{S P C} \mathcal{C}_{d^{\prime}}$ if and only if $d \geq d^{\prime}$ and $d \equiv d^{\prime}(\bmod 2)$.

Though the theorem easily follows from the previous theorem, we give an independent proof which explicitely constructs such a homomorphism. 
Proof: A homomorphism $\phi$ of $\mathcal{S P C} \mathcal{C}_{d+2}$ to $\mathcal{S P C _ { d }}$ can be defined as follows. If the last two coordinates of $v$ are 00 or 11 , then $\phi(v)$ is the restriction of $v$ to its first $d$ coordinates. Otherwise, to get $\phi(v)$, we first restrict $v$ to its first $d$ coordinates and then add the $d$-dimensional vector $J$. To see that $\phi$ is indeed a homomorphism of $\mathcal{S P C} \mathcal{C}_{d+2}$ to $\mathcal{S P C}$, one must resign $\mathcal{S P C _ { d + 2 }}$ at every vertex whose last two coordinates are 01 or 10. Associativity of homomorphisms then implies the existence of a homomorphism of $\mathcal{S P C _ { d }}$ to $\mathcal{S P C}_{d^{\prime}}$ when $d \geq d^{\prime}$ and $d \equiv d^{\prime}(\bmod 2)$. The inverse claim follows from Theorem 2.2 and the fact that every unbalanced cycle of $\mathcal{S P C} \mathcal{C}_{d}$ must have, in its image, an unbalanced cycle of $\mathcal{S P C} \mathcal{C}_{d^{\prime}}$.

\section{Folding lemma}

As mentioned before, it has been shown in [12] that the notion of signed homomorphisms on signed bipartite graphs already captures the notion of graph homomorphisms. The operations used to build this connection preserves planarity. Thus any homomorphism theory on planar graphs can be strengthened in the language of signed homomorphisms on planar signed bipartite graphs.

A key lemma in the study of homomorphism properties of a planar graph is the folding lemma of Klostermeyer and Zhang [9]. This lemma implies that for each planar graph $G$ of shortest odd cycle length $2 r+1$, and for each $k \leq r$, there is a planar homomorphic image $H$ of $G$ where every face of $H$ is of length $2 k+1$ and the shortest odd-length cycle of $H$ is also of length $2 k+1$. By considering unbalanced cycles instead of odd-length cycles, we will get the same result for the class of planar signed bipartite graphs.

Lemma 3.1 (Folding Lemma) Let $(G, \Sigma)$ be a planar signed bipartite graph of unbalanced girth $g$. If $C=v_{0} \cdots v_{r-1} v_{0}$ is a balanced facial cycle of $(G, \Sigma)$, or an unbalanced facial cycle of $(G, \Sigma)$ with $r>g$, then there is an integer $i \in\{0, \ldots, r-1\}$ such that the signed graph $\left(G^{\prime}, \Sigma_{G^{\prime}}\right)$ obtained from $(G, \Sigma)$ by identifying $v_{i-1}$ and $v_{i+1}$ (subscripts are taken modulo $r$ ) is a homomorphic image of $(G, \Sigma)$ of unbalanced girth $g$.

Proof: We follow notations and ideas of Section 4 in [9]. Suppose that $C=v_{0} \cdots v_{r-1} v_{0}$ is a balanced facial cycle of $(G, \Sigma)$, or an unbalanced facial cycle of $(G, \Sigma)$ with $r>g$. For each $i \in\{0, \ldots, r-1\}$, if $v_{i-1} v_{i} v_{i+1}$ does not belong to a $U C_{4}$ - which is always the case if $g>4-$ let $G_{i}$ be the graph obtained from $G$ by identifying $v_{i-1}$ and $v_{i+1}$, after having resigned at $v_{i-1}$ if $v_{i-1} v_{i}$ and $v_{i} v_{i+1}$ have opposite signs. If such a $G_{i}$ has unbalanced girth at least $g$ we are done (the mapping that identifies $v_{i-1}$ and $v_{i+1}$ is clearly a homomorphism of $(G, \Sigma)$ to $\left(G^{\prime}, \Sigma_{G^{\prime}}\right)=\left(G_{i}, \Sigma_{i}\right)$, where $\Sigma_{i}$ is the signature of $G_{i}$ induced by $\Sigma$ ). Otherwise (including the case $g=4$ ), it means that for each $i \in\{0, \ldots, r-1\}, G$ contains an unbalanced cycle $C_{i}$ of length $g$ passing through the segment $v_{i-1} v_{i} v_{i+1}$ of $C$. This kind of cycle is called a critical cycle of $(G, \Sigma)$ around $C$ containing $v_{i-1} v_{i} v_{i+1}$. Each critical cycle $C_{i}$ of length $g$ must contain a maximal segment $v_{\mu} C v_{\mu+p_{i}}=v_{\mu} v_{\mu+1} \cdots v_{\mu+p_{i}}$ with $v_{i-1} v_{i} v_{i+1} \subseteq v_{\mu} C v_{\mu+p_{i}}, v_{\mu-1} \notin C_{i}$ and $v_{\mu+p_{i}+1} \notin C_{i}$, where $p_{i}$ is called the pace of $C_{i}$ around $C$.

Let now $C_{\ell}$ be a critical cycle with the largest pace and $v_{b} C v_{d}$ be the maximal segment of $C$ contained in $C_{\ell}$. Consider another critical cycle $C_{b}$ that contains the segment $v_{b-1} v_{b} v_{b+1}$ and let $v_{a} C v_{c}$ be the maximal segment of $C$ contained in $C_{b}$ such that $v_{b-1} v_{b} v_{b+1} \subseteq v_{a} C v_{c}$. By the choice of $C_{\ell}, v_{c}$ must be contained in the segment $v_{b} C v_{d}$ and $v_{b} \neq v_{c} \neq v_{d}$. Note also that $v_{a} \neq v_{b}$. There are two possibilities: either $v_{a}$ is contained in the segment $v_{b} C v_{d}$ too or not. Let us first suppose the latter case (we will show later that the former case is not possible). Since $C$ is facial, no critical cycle intersects interior $(C)$. Thus, 


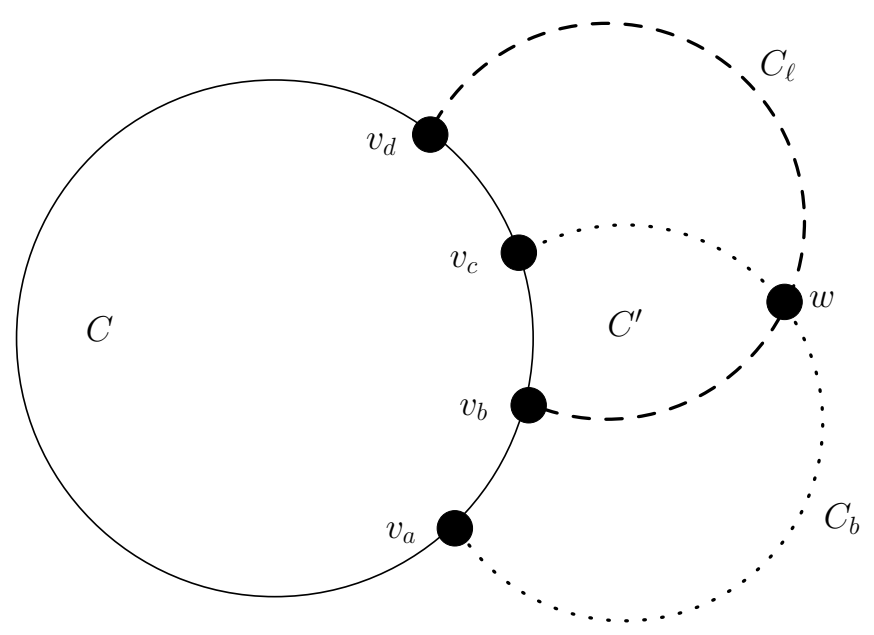

Fig. 3: Configuration for the proof of Lemma 3.1

since $v_{a}$ is not contained in the segment $v_{b} C v_{d}$, we get that $C_{\ell}$ and $C_{b}$ cross each other in exterior $(C)$ on some vertex, say $w$ (see Fig. 3). Moreover, $v_{a}, v_{b}, v_{c}$ and $v_{d}$ appear in this order around the facial cycle C.

Let $x_{0}-x_{1}-\cdots-x_{n-1}-x_{0}$ denote a signed Eulerian graph formed by the union of $\left(x_{i}, x_{i+1}\right)$-paths where $i \in \mathbb{Z}_{n}$. Then $C=v_{a}-v_{b}-v_{c}-v_{d}-v_{a}, C_{\ell}=v_{b}-v_{c}-v_{d}-w-v_{b}$ and $C_{b}=v_{a}-v_{b}-v_{c}-w-v_{a}$.

Let $C^{\prime}=v_{b}-v_{c}-w-v_{b}$, where $v_{b}-v_{c}$ is the path belonging to $C, v_{c}-w$ the path belonging to $C_{b}$ and $w-v_{b}$ the path belonging to $C_{\ell}$. We consider two cases.

1. The cycle $C^{\prime}$ is balanced. We then have:

(i) $v_{a}-v_{b}-w-v_{a}$, which is the symmetric difference of $C_{b}$ and $C^{\prime}$, is an unbalanced Eulerian graph, so it contains an unbalanced cycle. Since $C_{b}$ is critical, we get that $\left|v_{b}-w\right| \geq \mid v_{b}-$ $v_{c}|+| v_{c}-w \mid$, where $|x-y|$ is the length of the $(x, y)$-path; and

(ii) $v_{c}-v_{d}-w-v_{c}$, which is the symmetric difference of $C_{\ell}$ and $C^{\prime}$, is an unbalanced Eulerian graph, so it contains an unbalanced cycle. Since $C_{\ell}$ is critical, we get that $\left|v_{c}-w\right| \geq \mid v_{b}-$ $v_{c}|+| v_{b}-w \mid$.

By comparing (i) and (ii) we get that $\left|v_{b}-v_{c}\right| \leq 0$, a contradiction with the fact that $v_{b} \neq v_{c}$.

2. The cycle $C^{\prime}$ is unbalanced. We then have:

(i) $C^{\prime}$ contains an unbalanced cycle. Since $C_{b}$ is critical, we get that $\left|v_{c}-w\right| \geq\left|v_{c}-v_{d}\right|+\left|v_{d}-w\right|$.

(ii) $v_{a}-v_{b}-v_{c}-v_{d}-w-v_{a}$, which is the symmetric difference of $C_{\ell}$ and the symmetric difference of $C^{\prime}$ and $C_{b}$, is an unbalanced Eulerian graph, so it contains an unbalanced cycle. Since $C_{b}$ is critical, we get that $\left|v_{c}-w\right| \leq\left|v_{c}-v_{d}\right|+\left|v_{d}-w\right|$. 
By comparing (i) and (ii) we get that $\left|v_{c}-w\right|=\left|v_{c}-v_{d}\right|+\left|v_{d}-w\right|$ and the length of $v_{a}-v_{b}-$ $v_{c}-v_{d}-w-v_{a}$ is the same as the length of the critical cycle $C_{b}$. Thus $v_{a}-v_{b}-v_{c}-v_{d}-w-v_{a}$ itself is critical but with a pace larger than the pace of $C_{\ell}$, a contradiction.

It remains to show that $v_{a}$ cannot be contained in the segment $v_{b} C v_{d}$. Suppose to the contrary that $v_{a}$ is contained in the segment $v_{b} C v_{d}$, possibly with $v_{a}=v_{d}$. Since $C_{\ell}$ is with a largest pace $p$, we conclude that $2 p>|V(C)|$, as otherwise $C_{\ell}$ would be of larger pace. We distinguish two cases.

1. The cycle $C$ is balanced. Then the symmetric difference of $C_{\ell}$ and $C$ is an unbalanced Eulerian graph that contains an unbalanced cycle of length shorter than the length of the critical cycle $C_{\ell}$, a contradiction.

2. The cycle $C$ is unbalanced. Since the length $\ell(C)$ of $C$ is different from $g$, we get $\ell(C) \geq g+2$; in particular, $\ell(C)>\ell\left(C_{b}\right)$. We consider the symmetric difference of $C_{b}$ and the symmetric difference of $C_{\ell}$ and $C$. The result is an unbalanced Eulerian graph that contains an unbalanced cycle with a length shorter than the length of the critical cycle $C_{\ell}$, since the $\left(v_{c}, v_{a}\right)$-path of $C_{\ell}$ belonging to $C$ is replaced by a shorter $\left(v_{c}, v_{a}\right)$-path belonging to $C_{b}$, again a contradiction.

We thus get that there is some $G_{i}$ such that $\left(G_{i}, \Sigma_{i}\right)$ is the required signed graph.

By repeated application of this lemma we get the following:

Corollary 3.2 Given a planar signed bipartite graph $(G, \Sigma)$ of unbalanced girth $g$, there is a homomorphic image $\left(G^{\prime}, \Sigma^{\prime}\right)$ of $(G, \Sigma)$ such that:

- $G^{\prime}$ is planar,

- $\left(G^{\prime}, \Sigma^{\prime}\right)$ is a signed bipartite graph,

- $\left(G^{\prime}, \Sigma^{\prime}\right)$ is of unbalanced girth $g$,

- every face of $\left(G^{\prime}, \Sigma^{\prime}\right)$ is an unbalanced cycle of length $g$.

Proof: We can assume that $G$ is connected (otherwise, we may pick one vertex in each component and identify them). If $u$ is a cut-vertex of $(G, \Sigma)$, with two neighbors $v_{1}$ and $v_{2}$ lying on the outerface and not belonging to the same block, the signed graph $\left(G_{1}, \Sigma_{1}\right)$, obtained by identifying $v_{1}$ and $v_{2}$ (after having resigned at $v_{1}$ if necessary), is clearly a bipartite homomorphic image of $(G, \Sigma)$ with unbalanced girth $g$. Repeating this procedure for every cut-vertex of $(G, \Sigma)$, we get a 2-connected signed bipartite graph, say $\left(G_{k}, \Sigma_{k}\right)$, which is a homomorphic image of $(G, \Sigma)$ with unbalanced girth $g$. Every face of $\left(G_{k}, \Sigma_{k}\right)$ is then either a balanced cycle or an unbalanced cycle of length at least $g$. We can then apply Lemma 3.1 until every face is an unbalanced cycle of length $g$, and get the desired result. 


\section{An extension of the Four-Color Theorem}

In this section, we prove Theorem 1.6.

Proof: First assume that every planar signed bipartite graph of unbalanced girth at least $2 k$ admits a homomorphism to $S P C_{2 k-1}$ and let $G$ be a planar $2 k$-regular multigraph with no odd edge-cut of less than $2 k$ edges. Using Tutte's matching theorem we can easily verify that $G$ admits a perfect matching. Let $M$ be a perfect matching of $G$. Let $G^{D}$ be the dual of $G$ with respect to some embedding of $G$ on the plane. Since $G$ is $2 k$-regular, $G^{D}$ is clearly bipartite. Let $M^{D}$ be the edges in $G^{D}$ corresponding to the edges of $M$. It is now easy to check that $\left(G^{D}, M^{D}\right)$ is a planar signed bipartite graph of unbalanced girth $2 k$. Therefore, by our main assumption, $\left(G^{D}, M^{D}\right)$ admits a homomorphism to $\mathcal{S P} \mathcal{C}_{2 k-1}$. This mapping induces a $2 k$-edge-coloring on $G^{D}$ (not necessarily a proper edge-coloring) using colors $e_{1}, \ldots, e_{2 k-1}, J$. By Theorem 2.2 every unbalanced cycle has received exactly $2 k$ different colors. In particular each face of $G^{D}$, which is an unbalanced cycle of length $2 k$, has received all $2 k$ colors. Thus reassigning these colors to their corresponding edges in $G$ will result in a proper $2 k$-edge-coloring of $G$.

Now we assume that every planar $2 k$-regular multigraph with no odd edge-cut of less than $2 k$ edges is (properly) $2 k$-edge-colorable. Let $(G, \Sigma)$ be a plane signed bipartite graph of unbalanced girth $2 k$. We would like to prove that this signed graph admits a homomorphism to $\mathcal{S P C} \mathcal{C}_{2 k-1}$. By Corollary 3.2 we may assume that each face of $(G, \Sigma)$ is an unbalanced cycle of length exactly $2 k$. Let $G^{D}$ be the dual of $G$ with respect to its embedding on the plane. Obviously $G^{D}$ is a $2 k$-regular multigraph, furthermore it is easy to check that $G^{D}$ has no odd edge-cut of strictly less than $2 k$ edges (this is the dual of having unbalanced girth at least $2 k$ ). Thus, by our main assumption, $G^{D}$ is $2 k$-edge colorable. Let $M_{i}$ be one of the color classes, which, therefore, is a perfect matching. Let $\Sigma_{i}$ be the edges of $G$ corresponding to the edges of $G^{D}$ in $M_{i}$. We first claim that $\Sigma_{i}$ is equivalent to $\Sigma$. This is the case because in both $\left(G, \Sigma_{i}\right)$ and $(G, \Sigma)$ each face is an unbalanced cycle, and any other cycle is unbalanced if and only if it bounds an odd number of faces. That means that the sets of unbalanced cycles in both signatures are the same and the claim follows by Theorem 1.2. To complete the proof note that we have partitioned edges of $G$ into $2 k$ sets $\Sigma_{i}$ each being a signature of $(G, \Sigma)$. Thus, by Theorem $2.4,(G, \Sigma)$ admits a homomorphism to $\mathcal{S P C}_{2 k-1}$.

Since Seymour's conjecture is verified up to $k \leq 8$, see [6], [3], [4] and [2], we conclude that:

Corollary 4.1 Every planar signed bipartite graph of unbalanced girth 4 (6 and 8, respectively) admits a homomorphism to $\mathcal{S} \mathcal{P C}_{3}\left(\mathcal{S P C}_{5}, \mathcal{S P C}\right.$, respectively).

Note that if $G$ is a simple bipartite graph, then the unbalanced girth of $(G, \Sigma)$ is at least 4 . Furthermore, note that $\mathcal{S P C}_{3}$ is isomorphic to $\left(K_{4,4}, M\right)$ where $M$ is a perfect matching of $K_{4,4}$. Therefore:

Corollary 4.2 Every planar signed bipartite graph admits a homomorphism to $\left(K_{4,4}, M\right)$.

Using Theorem 6.2 of [12] it follows that this corollary is stronger than the Four-Color Theorem. A fact that, in the edge-coloring formulation, was already proved by P. Seymour [13].

\section{Remarks}

1. B. Guenin [5] conjectured that in Conjecture 1.5 the condition of planarity can be replaced with the weaker condition of having no $\left(K_{5}, E\left(K_{5}\right)\right)$ as a minor, which, if true, would imply the same results for a larger class. 
2. If Conjecture 1.5 holds, i.e., if every planar signed bipartite graph or planar odd signed graph of unbalanced girth $g$ admits a homomorphism to a signed projective cube of unbalanced girth $g$, then, by Theorem 2.5, any such planar signed graph also admits a homomorphism into projective cubes of unbalanced girth $g-2 i$.

We believe that for this latter case, when $i \geq 1$, not all vertices of the signed projective cube are needed. Indeed it is shown in [11] that, for planar odd signed graphs, determining minimal subgraphs of the signed projective cube $\mathcal{S P \mathcal { C } _ { 2 g }}$ that would bound the class of planar odd signed graphs of unbalanced girth at least $2 k+1, k \geq g$, would relate to questions such as determining the supremum of the fractional and circular chromatic numbers of planar graphs of given odd girth.

We believe an analog question for the case of signed bipartite graph would result in development of further theories and discovery of signed bipartite graphs with high symmetries. Thus we ask:

Problem 5.1 What are the minimal subgraphs of $\mathcal{S} \mathcal{P C}_{2 g-1}$ to which every planar signed bipartite graph of unbalanced girth $2 g+2 i, i \geq 1$, admits a homomorphism?

A particular case of this question, which is the bipartite analog of Grötzsch's theorem and JaegerZhang's conjecture, is studied in [1].

\section{Acknowledgements}

We would like to acknowledge support from CNRS (France) through the PEPS project HOGRASI and partial support by APVV, Project 0223-10 (Slovakia).

\section{References}

[1] C. Charpentier, N. Naserasr and E. Sopena. Extensions of Grötzsch's theorem and Jaeger-Zhang's conjecture to planar signed bipartite graphs. In preparation.

[2] M. Chudnovsky, K. Edwards and P. Seymour. Edge-colouring eight-regular planar graphs. Manuscript (2012), available at http://arxiv.org/abs/1209.1176.

[3] Z. Dvořák, K. Kawarabayashi and D. Král’. Packing six T-joins in plane graphs. Manuscript (2010), available at http://arxiv.org/abs/1009.5912.

[4] K. Edwards. Optimization and Packings of T-joins and T-cuts. M.Sc. Thesis, McGill University (2011).

[5] B. Guenin. Packing odd circuit covers: A conjecture, manuscript.

[6] B. Guenin. Packing T-joins and edge-colouring in planar graphs. Mathematics of Operations Research, to appear.

[7] T.R. Jensen and B. Toft. Graph Coloring Problems. Wiley-Interscience Series in Discrete Math. and Optim. (1995).

[8] P. Hell, J. Nešetřil. Graphs and homomorphisms. Oxford Lecture Series in Mathematics and its Applications, 28. Oxford University Press, Oxford, 2004. 


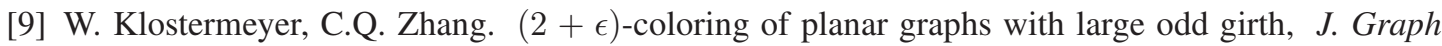
Theory 33 (2) (2000), 109-119.

[10] R. Naserasr. Homomorphisms and edge-colourings of planar graphs. J. Combin. Theory Ser. B 97 (2007), no. 3, 394-400.

[11] R. Naserasr. Mapping planar graphs into projective cubes. J. Graph theory, to appear.

[12] R. Naserasr, E. Rollová and E. Sopena. Homomorphisms of signed graphs, submitted. Available at http://www.labri.fr/perso/sopena/Publications.

[13] P. Seymour. Matroids, Hypergraphs and the Max.-Flow Min.-Cut Theorem. D. Phil. Thesis, Oxford (1975), page 34.

[14] T. Zaslavsky. Signed graphs. Discrete Applied Math. 4(1) (1982), 47-74. 